\title{
Pharmacologic relaxation of vein grafts is beneficial compared with pressure distention caused by upregulation of endothelial nitric oxide synthase and nitric oxide production
}

Ada W. Y. Chung, PhD, ${ }^{a}$ Pooja Rauniyar, BSc, ${ }^{a}$ Honglin Luo, MD, PhD, ${ }^{\text {a }}$ York N. Hsiang, MD, ${ }^{\text {b }}$ Cornelis van Breemen, DVM, $\mathrm{PhD}^{\mathrm{a}}$ and Elena B. Okon, $\mathrm{PhD}^{\mathrm{a}}$

From The James Hogg iCAPTURE Center for the Cardiovascular and Pulmonary Research, ${ }^{a}$ St Paul's Hospital, Vancouver, British Columbia, Canada, and the Department of Surgery, ${ }^{\mathrm{b}}$ University of British Columbia, Vancouver, British Columbia, Canada.

The study was supported by a Grant-in-Aid from the Heart and Stroke Foundation of British Columbia and Yukon. A. C. is the recipient of Heart and Stroke Foundation of Canada/AstraZeneca Canada Research Fellowship Award. H. L. is a New Investigator of the CIHR/St Paul's Hospital Foundation Award and a Scholar of the Michael Smith Foundation for Health Research.

Received for publication Feb 6, 2006; revisions received March 30, 2006; accepted for publication April 24, 2006.

Address for reprints: Ada W. Y. Chung, $\mathrm{PhD}$, Room 2099, Cardiovascular Sciences, 950 28th W Ave, Vancouver, British Columbia, V5Z 4H4, Canada (E-mail: achung@ mrl.ubc.ca).

J Thorac Cardiovasc Surg 2006;132:925-32

$0022-5223 / \$ 32.00$

Copyright () 2006 by The American Association for Thoracic Surgery

doi:10.1016/j.jtcvs.2006.04.033
Objective: Pressure distention of veins during preparation for bypass surgery is believed to impair vascular integrity and reduce graft patency. We previously suggested a combination of pharmacologic vasodilatators as an alternative to distention. Vascular homeostasis is largely regulated by nitric oxide. We investigated the role of distention in comparison with pharmacologic vasorelaxation in the regulation of nitric oxide synthases, nitric oxide bioavailability, and vascular reactivity in vein grafts.

Methods: In a porcine model the internal jugular vein from either side received pressure distention or the combination of vasodilators ( $\alpha$-adrenergic antagonist, phenoxybenzamine, $10 \mu \mathrm{mol} / \mathrm{L}$; Rho-kinase inhibitor, HA-1077 [fasudil], 50 $\mu \mathrm{mol} / \mathrm{L}$; calcium blocker, nicardipine, $1 \mu \mathrm{mol} / \mathrm{L}$ ) and then was grafted into the carotid artery. Regulation of nitric oxide synthase, as well as nitrate and nitrite levels, were examined in vein grafts after 2 weeks of implantation.

Results: Distention of jugular veins resulted in reduction of vasoconstriction in response to depolarization and agonist stimulation. Arterial grafting doubled inducible nitric oxide synthase expression in both grafts but caused a pronounced upregulation of endothelial nitric oxide synthase protein (by $57.3 \% \pm 5 \%$ ) only in drug-treated grafts, whereas in distended grafts the endothelial nitric oxide synthase level was decreased by $27.5 \% \pm 2.7 \%$. The downregulated endothelial nitric oxide synthase level in the distended grafts was accompanied by a $45.2 \% \pm 3.1 \%$ reduction of phospho-endothelial nitric oxide synthase $\operatorname{Ser}^{1177}$ levels and by a significant reduction in nitric oxide synthase activity $(12.1 \% \pm 1.2 \%)$ and nitrate production $(48.9 \% \pm 5.6 \%)$ in comparison with that seen in drug-treated grafts.

Conclusions: Pharmacologic preparation of the vein grafts results in upregulation of endothelial nitric oxide synthase and increased nitric oxide production in the vein grafts after arterial implantation. This might provide greater clinical benefit than conventional pressure-distention methods.

$\mathrm{T}$ The saphenous vein (SV) is a commonly used conduit for cardiac and peripheral revascularization. Because of its long length and the ease of harvesting, the demand for SVs in grafting remains high. However, it is spasmodic and is routinely distended during preparation for grafting to overcome vasospasm. This procedure might result in lower vein graft patency. ${ }^{1,2}$

High pressure-distention immediately and irreversibly impaired vascular contractility and endothelial function ${ }^{3,4}$ resulting in reduced bioavailability of nitric oxide (NO), ${ }^{5}$ and endothelial and medial NO synthases (NOSs), ${ }^{6}$ and a decrease in smooth muscle cell (SMC) response to relaxant signaling. ${ }^{7}$ Serious damage of the 


\author{
Abbreviations and Acronyms \\ eNOS $=$ endothelial nitric oxide synthase \\ iNOS $=$ inducible nitric oxide synthase \\ $\mathrm{NO}=$ nitric oxide \\ NOS = nitric oxide synthase \\ SMC $=$ smooth muscle cell \\ $\mathrm{SV}=$ saphenous vein
}

endothelial lining after manual flushing and distention has been found in earlier investigations. ${ }^{8,9}$ Distended veins also exhibited increased platelet and leukocyte adhesion, ${ }^{10}$ excessive extracellular matrix accumulation in the neointima, ${ }^{11}$ and activation of proliferation signaling (unpublished data), underlying the accelerated development of neointima. ${ }^{10}$

$\mathrm{NO}$ is crucial in regulating graft remodeling not only for its role in control of blood flow, but also for its inhibitory effects on SMC proliferation and adhesion and its proangiogenic $^{12}$ role, which alleviates the consequences of ischemia. In the vasculature NO is mostly synthesized by the constitutively expressed endothelial NOS (eNOS) and the inducible NOS (iNOS). Hence the production of NO has been found to be dysfunctional after balloon injury and in vein grafts when vascular SMC proliferation and neointima formation are progressing, and a deficit of NO has been implicated in decreased proliferative modification of vascular SMC. ${ }^{13-15}$ Therefore, downregulation of NOS and the consequent reduced NO production resulting from pressure distention of vein grafts could contribute to thrombosis and neointima development. However, the effects of distention on the regulation of NOS in vein grafts are less studied.

Because of the high susceptibility of human SVs to vasoconstriction, ${ }^{3,4}$ pressure distension during surgical preparation for grafting cannot be abolished without replacement with an alternative procedure to prevent vasospasm. We have suggested the topical application of a combination of effective pharmacologic vasodilatators as an alternative to pressure distention. ${ }^{4,11}$ In the present study we investigated the effect of pressure distention compared with pharmacologic vasorelaxation of vein grafts on the regulation of NOS and the production of NO in a porcine vein graft model. We hypothesized that pressure distention of the veins compromised vascular integrity and the NO system by causing downregulation of NOS (Figure 1).

\section{Materials and Methods}

\section{Porcine Bypass Grafting}

All animal procedures were approved by the University of British Columbia Animal Care Committee. Reversed segments of jugular veins were grafted into the carotid arteries of 8 female white pigs (weight, 60-65 kg). Pigs were given a general anesthetic (a single
A

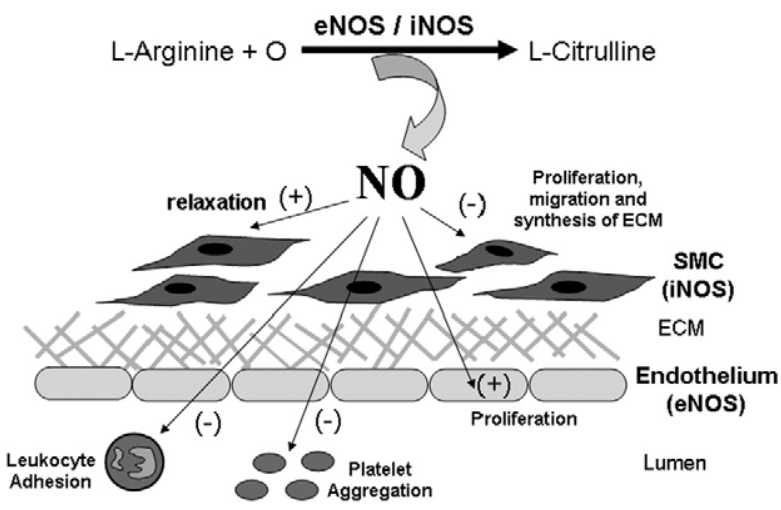

B

Experiment Hypothesis:

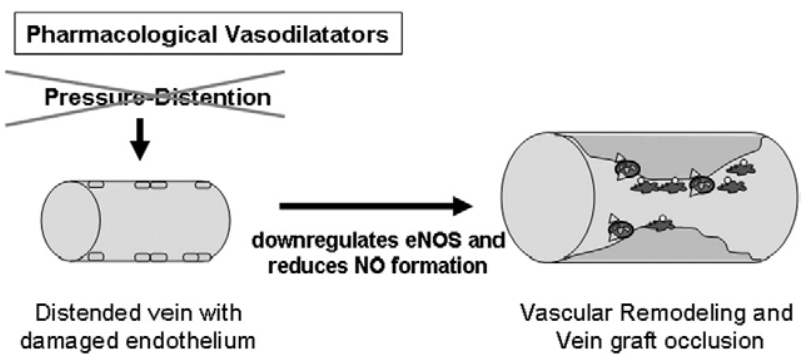

Figure 1. A, An illustration showing the beneficial effects of nitric oxide (NO). In the vasculature NO is generated from L-arginine by both constitutive and inducible NO synthase (endothelial NO synthase (eNOS) in endothelium and inducible NO synthase (iNOS) in smooth muscle cells (SMCs)). NO exerts its protective effects by inducing smooth muscle relaxation; inhibiting SMC proliferation, migration, and synthesis of extracellular matrix (ECM); inhibiting platelet and leukocyte activation and adhesion; and stimulating endothelial cells proliferation. B, We hypothesize that the replacement of pressure distention with pharmacologic vasodilatators in vein grafting preserves endothelium and NO formation in the vein graft. The augmented bioavailability of NO would be beneficial to suppress vascular remodeling and vein graft occlusion.

dose of ketamine, 800-1400 mg), and under sterile conditions, a midline neck incision was made to expose both the common carotid and internal jugular veins. Six-centimeter segments of the veins were dissected from each side and randomized to receive pressure distension or immersion in a vasodilatory solution. Veins randomized to pressure distension were distended with physiologic solution at $300 \mathrm{~mm} \mathrm{Hg}$ for 2 minutes, and a segment was cut as a distended vein sample. From the vein randomized to the vasodilatory solution, a piece $(4 \mathrm{~mm})$ was cut as a nondistended vein sample, and the rest was placed in a bath containing a combination of vasodilatatory drugs ( $\alpha$-adrenergic antagonist, phenoxybenzamine, $10 \mu \mathrm{mol} / \mathrm{L}$; Rho-kinase inhibitor, HA-1077 [fasudil], 50 $\mu \mathrm{mol} / \mathrm{L}$; calcium blocker, nicardipine, $1 \mu \mathrm{mol} / \mathrm{L}$ ) for $30 \mathrm{~min}-$ 
utes. ${ }^{4,11}$ After either pressure distension or a vasodilatory bath, the veins were grafted to the divided carotid artery by using an end-to-end anastomotic technique as an interposition vein graft. After 2 weeks, the 7 pigs that survived the operation were given another general anesthetic, and the interposition vein grafts were harvested. The drug-treated graft was considered as a control for damaging pressure distention. All animals were then killed.

\section{Histology \\ Representative segments were formalin fixed and embedded in paraffin, and 3- $\mu \mathrm{m}$ cross-sections were prepared and stained with antibodies against von Willebrand factor (dilution 1:400), which label endothelium and allow us to evaluate its thickness and integrity, and with Movat pentachrome, which labels SMCs, elas- tin, and other extracellular matrix components in the vessels. Image acquisition and processing was performed with a Nikon MicroPhot microscope (Nikon Inc, Garden City, NY), and images were captured by using a SPOT digital camera (Diagnostic Instru- ment Inc, Sterling Heights, Mich).}

\section{Vasomotor Function and Elasticity}

For studying vasomotor function, 3-mm vein segments were suspended between 2 stainless-steel clips, placed for 2 hours in glass-jacketed tissue baths containing oxygenated physiologic buffer saline $(\mathrm{pH} 7.4)$ at $37^{\circ} \mathrm{C}$, and equilibrated as described previously. ${ }^{3}$ One of the clips was connected to a force transducer, and the force was registered with A/S Myotechnology Myograph (Aarhus N, Denmark). The contraction of the vessels was stimulated with $50 \mu \mathrm{mol} / \mathrm{L}$ phenylephrine.

Endothelium-dependent relaxation was studied, with acetylcholine $(10 \mathrm{nmol} / \mathrm{L}$ to $3 \mu \mathrm{mol} / \mathrm{L}$, median effective concentration $=$ $100 \mathrm{nmol} / \mathrm{L}$ ) added in a cumulative manner to the precontracted veins.

The vessel elasticity was elucidated from the length-tension curves, which were obtained by measuring the passive tension while gradually increasing the circumferential length of the vessel segments. Data acquisition and analysis were as previously described. ${ }^{3,4}$

\section{Reverse Transcription Polymerase Chain Reaction}

Total RNA was extracted from the flash-frozen samples by using TRIzol reagent (Invitrogen Life Technologies, Carlsbad, Calif). ${ }^{11}$ RNA $(1 \mu \mathrm{g})$ was subjected to reverse transcription by using Moloney murine leukemia virus reverse transcriptase, ribonuclease inhibitor, and random primers (Invitrogen). ${ }^{11}$ Reverse transcription reaction mixture $(5 \mu \mathrm{L})$ was used in the polymerase chain reaction experiments with the addition of AmpliTaq Gold DNA polymerase (Applied Biosystems, Foster City, Calif) and gene-specific primers for eNOS (forward: CAG-TGT-CCA-ACATGC-TGC-TGG-AAA-TTG, reverse: TAA-AGG-TCT-TCT-TCCTGG-TGA-TGC-C, 485 bp) and iNOS (forward: GGC-CTC-GCTCTG-GAA-AGA, reverse: TCC-ATG-CAG-ACA-ACC-TT, 499 $\mathrm{bp})$. The reference gene $\beta$-actin, which is expressed in vascular cell types (forward: CAG-GCA-CCA-GGG-CGT, reverse: ATGGCT-GGG-GTG-TTG-AAG, 282 bp) served as a control to ensure equal sample loading (NAPS Unit Oligonucleotide Syn- thesis Laboratory, Biotechnology Laboratory, The University of British Columbia).

\section{Western Immunoblotting}

Flash-frozen segments were ground with liquid nitrogen in a stainless-steel mortar and pestle. Tissue powder was mixed in 9 volumes of ice-cold lysis buffer $(50 \mathrm{mmol} / \mathrm{L}$ Tris- $\mathrm{HCl}, \mathrm{pH} \mathrm{7.4}$, with $3.1 \mathrm{mmol} / \mathrm{L}$ sucrose, $1 \mathrm{mmol} / \mathrm{L}$ dithiothreitol, $10 \mu \mathrm{g} / \mathrm{mL}$ leupeptin, $10 \mu \mathrm{g} / \mathrm{mL}$ soybean trypsin inhibitor, $2 \mu \mathrm{g} / \mathrm{mL}$ aprotinin, and $0.1 \%$ Triton $\mathrm{X}-100$ ). After 20 minutes of incubation on ice, samples were homogenized with a glass homogenizer. Protein samples $(10 \mu \mathrm{g})$ were separated on a $7 \%$ sodium dodecylsulfatepolyacrylamide gel electrophoresis and transferred to polyvinyl difluoride membranes (Biorad, Hercules, Calif). ${ }^{11}$ Membranes were incubated with mouse monoclonal anti-eNOS, anti-iNOS (dilution 1:1000; BD Biosciences, Mississauga, Ontario, Canada), or rabbit polyclonal anti-phospho-eNOS $\mathrm{Ser}^{1177}$ (dilution 1:1000; Cell Signaling, Beverly, Mass) antibodies for 2 hours, followed by horseradish peroxidase-labeled secondary anti-mouse or antirabbit IgG antibodies (dilution 1:2500) for 1 hour. Immunoreactive protein was visualized by using an enhanced chemiluminescence kit (Amersham Life Sciences, Buckinghamshire, United Kingdom).

\section{Measurement of NOS Activity and Concentrations of Nitrate and Nitrite}

NO concentration and activity of eNOS and iNOS in protein extract $(30 \mu \mathrm{g})$ was determined colorimetrically by using an NOS assay kit (Calbiochem, San Diego, Calif). NO was assayed by measuring the sum of concentrations of nitrate $\left(\mathrm{NO}_{3}{ }^{-}\right)$and nitrite $\left(\mathrm{NO}_{2}{ }^{-}\right)$, the oxidation products of $\mathrm{NO}$, which were quantified colorimetrically relative to a nitrate standard curve.

\section{Materials}

All other reagents were of the highest molecular grade and purchased from Sigma (St Louis, Mo), unless otherwise stated.

\section{Statistics}

Data were reported as means \pm standard error of the mean from 8 independent experiments. Statistical analysis was performed with GraphPad Prism software (San Diego, Calif). Differences between groups were studied by means of 1-way analysis of variance.

\section{Results}

\section{Vasomotor Function, Morphology, and}

\section{Vessel Elasticity}

Distention irreversibly decreased the contractile response of the jugular vein to $\alpha$-adrenergic stimulation and depolarization to about $10 \%$ of the original value and abolished vasorelaxation in response to acetylcholine (Figure 2, A). The presence of the endothelial layer, on average, was not changed significantly (Figure 3,A-C), although on some cross-sections endothelial cells appeared to be separated from each other. On Movat-stained sections, elastin (the black fibril structure) was present throughout the media as continuous circumferentially oriented bands, which separated SMC layers (Figure 3, $D$ and $E$ ). Distention disrupted 

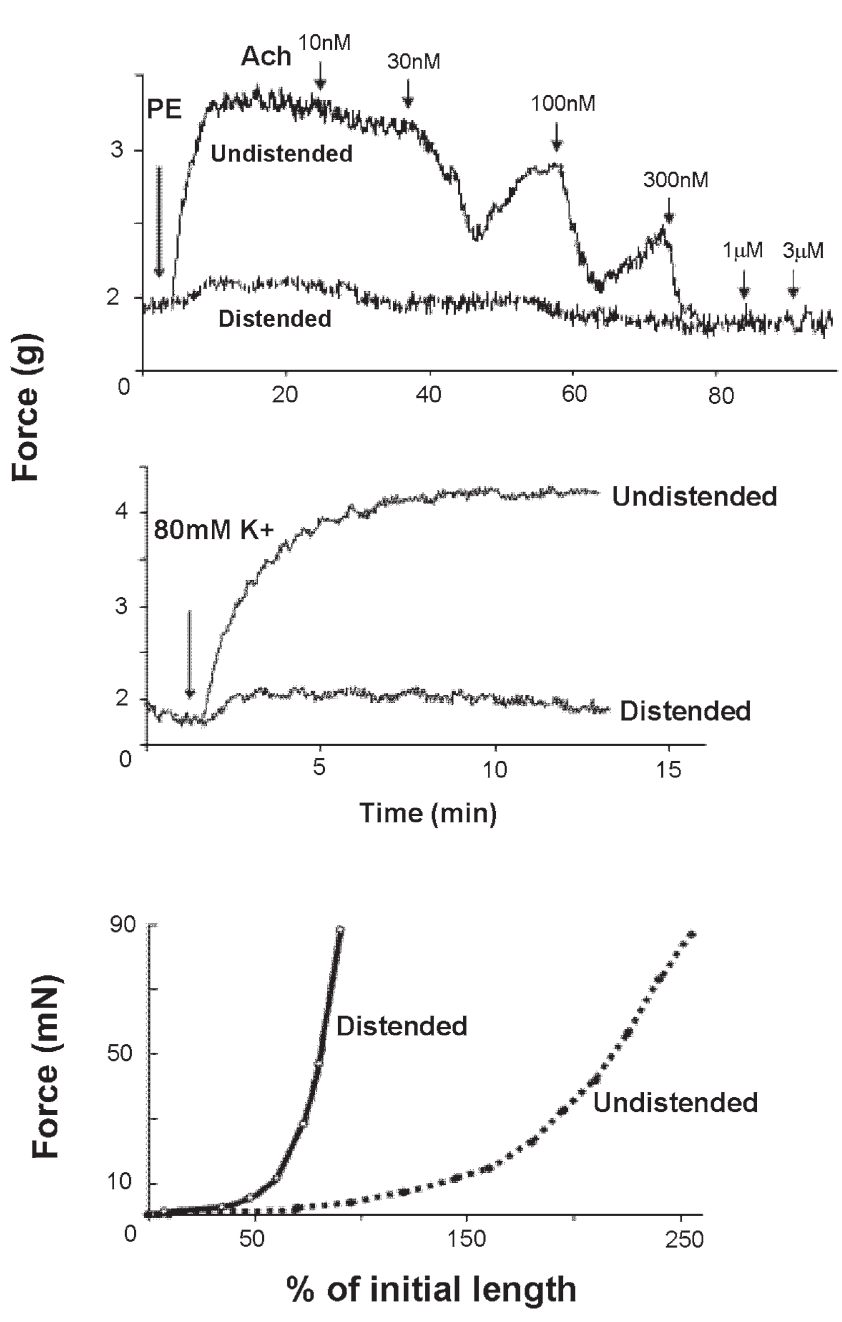

Figure 2. Vasomotor properties. A, Contraction of porcine undistended and distended veins in response to $\alpha$-adrenergic stimulation (50 $\mu \mathrm{mol} / \mathrm{L}$ phenylephrine [PE]) and acetylcholine (Ach)induced relaxation $(10 \mathrm{nmol} / \mathrm{L}$ to $3 \mu \mathrm{mol} / \mathrm{L})$ and contraction induced by depolarization $\left(80 \mathrm{mmol} / \mathrm{L} \mathrm{K}^{+}\right)$. B, Length-tension curves of an undistended (dotted line) and a distended (solid line) porcine vein. Results are representative of 6 similar experiments.

the organized fibril structure of elastin, which appeared as discontinuous elastin fragments (Figure $3, E$ ). The effect of distention on the elastic properties studied in parallel showed that the distended vein markedly lost elasticity (Figure 2, B).

\section{Regulation of NOS Expression and Activity}

Neither distention nor arterial implantation modified eNOS gene transcription in vein grafts (Figure 4,A), whereas iNOS transcription, not detected before grafting, was markedly upregulated in both postgrafts (Figure 4).

Although pressure distention did not instantly affect the protein expression of eNOS, different protein levels were observed in 2 postgrafts. In comparison with the pregrafted
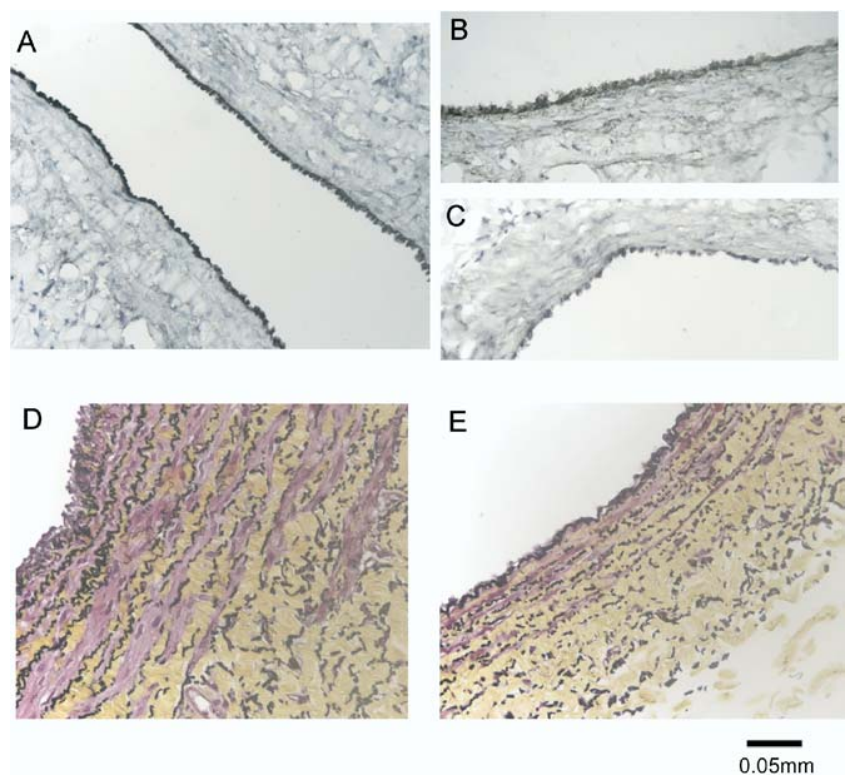

Figure 3. von Willebrand factor-stained cross-sections from undistended (control, A) and distended (B and C) veins indicating the presence of endothelial cells. Movat-stained sections showing the elastin fibril structure (black) in the undistended (D) and distended (E) veins. Scale bars $=0.05 \mathrm{~mm}$. Representative images from 7 to 8 independent experiments are shown.

level, eNOS expression in drug-treated grafts was increased by $57.3 \% \pm 5.0 \%$, whereas in distended grafts it was decreased by $27.5 \% \pm 2.7 \%$. eNOS enzymatic activity was enhanced by phosphorylation at $\mathrm{Ser}^{1177}$, and its phosphorylation level was highly upregulated by $110 \% \pm 18 \%$ in the drug-treated grafts. Phosphorylation of eNOS in the distended grafts remained at the pregrafted level. Thus the eNOS protein expression and phosphorylation levels in the distended postgrafts were about half of those in the drugtreated grafts (Figure 5, $A$ and $C$ ).

The iNOS protein levels in both veins were approximately doubled after grafting (Figure 5, B). Both postgrafts demonstrated increased NOS activity, but the drug-treated grafts had a significantly higher NOS activity than the distended grafts by $12.1 \% \pm 1.2 \%$ (Figure 6).

\section{Regulation of NO Production}

$\mathrm{NO}$ is readily oxidized to form $\mathrm{NO}_{3}{ }^{-}$and $\mathrm{NO}_{2}{ }^{-}$, and therefore $\mathrm{NO}$ production was measured as the sum of these oxidation products. Arterial implantation differentially regulated NO production in 2 grafts, with NO production being increased in drug-treated grafts by $20.3 \% \pm 4.4 \%$ but reduced in distended grafts by $17.0 \% \pm 3.2 \%$. Because distention had a small inhibitory effect on the pregrafted vein, the NO production in the drug-treated grafts was $48.9 \% \pm 5.6 \%$ higher than in the distended grafts (Figure 6). 


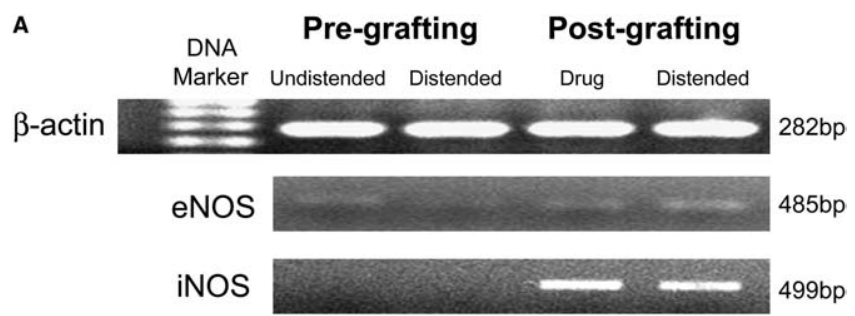

B

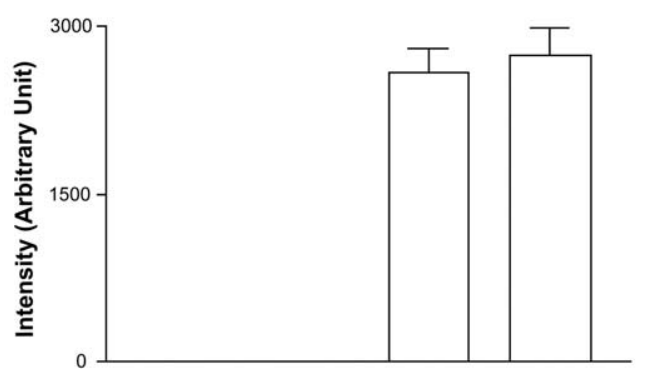

Figure 4. Regulation of gene transcription of endothelial nitric oxide synthase (eNOS) and inducible nitric oxide synthase (iNOS) in vein grafts prepared by pressure distention and pharmacologic vasodilatators. A, Representative polymerase chain reaction results showing the mRNA transcription of eNOS, iNOS, and $\boldsymbol{\beta}$-actin (loading control). B, Bar chart is the densitometric measurement of iNOS transcription.

\section{Discussion}

The present study reveals the effects of pressure distention in comparison with pharmacologic vasodilatation and arterial hemodynamics on the regulation of NOS in a porcine vein graft model. After 2 weeks of implantation into the arterial circulation, eNOS expression and phosphorylation were markedly less in the distended grafts compared with the drug-treated ones and were associated with lower levels of NOS activity and NO production. The inducible enzyme iNOS, which was undetectable in the veins before grafting, was equally induced during implantation in both types of grafts but did not compensate for the reduced NO generation in the vascular tissue of the distended grafts.

Distention with moderate pressure (eg, $300 \mathrm{~mm} \mathrm{Hg}$ ) drastically affected the veins both structurally and functionally. It overstretched the veins, and as a result, the vessels irreversibly lost their contractile ability and passive elasticity. The loss of the contractile responses to depolarization with $80 \mathrm{mmol} / \mathrm{L} \mathrm{K}^{+}$and to the $\alpha$-adrenergic receptor agonist phenylephrine demonstrates the impairment of the SMCs (Figure 2, A). The loss of elasticity could be due to both damage to the SMCs and the fracture of the elastin fibrillar structure (Figure 2,B). ${ }^{16}$

Denudation of the endothelial cells was not observed at this pressure. Although some von Willebrand factor-stained slides of the distended veins showed discontinuity in the
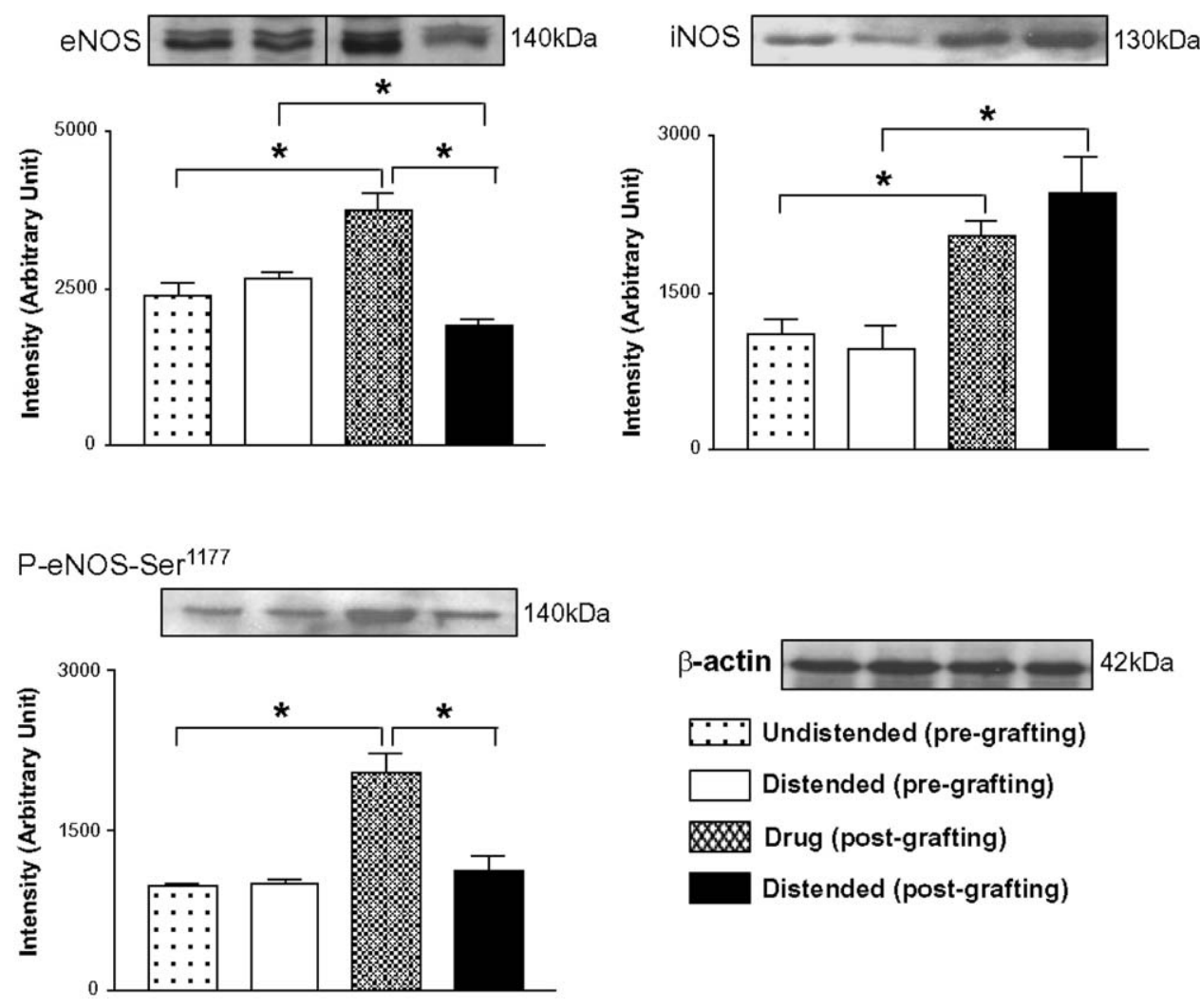

Figure 5. Regulation of nitric oxide synthase expression in vein grafts prepared by pressure distention and pharmacologic vasodilatators. Densitometric measurement of protein expressions of endothelial nitric oxide synthase (eNOS; A), inducible nitric oxide synthase (iNOS; B), and phospho-eNOS Ser ${ }^{1177}$ (C) is shown. Insets, representative immunoblots showing eNOS, iNOS phospho-eNOS Ser ${ }^{1177}$, and $\beta$-actin immunoreactivity. ${ }^{*} \boldsymbol{P}<.05$. 

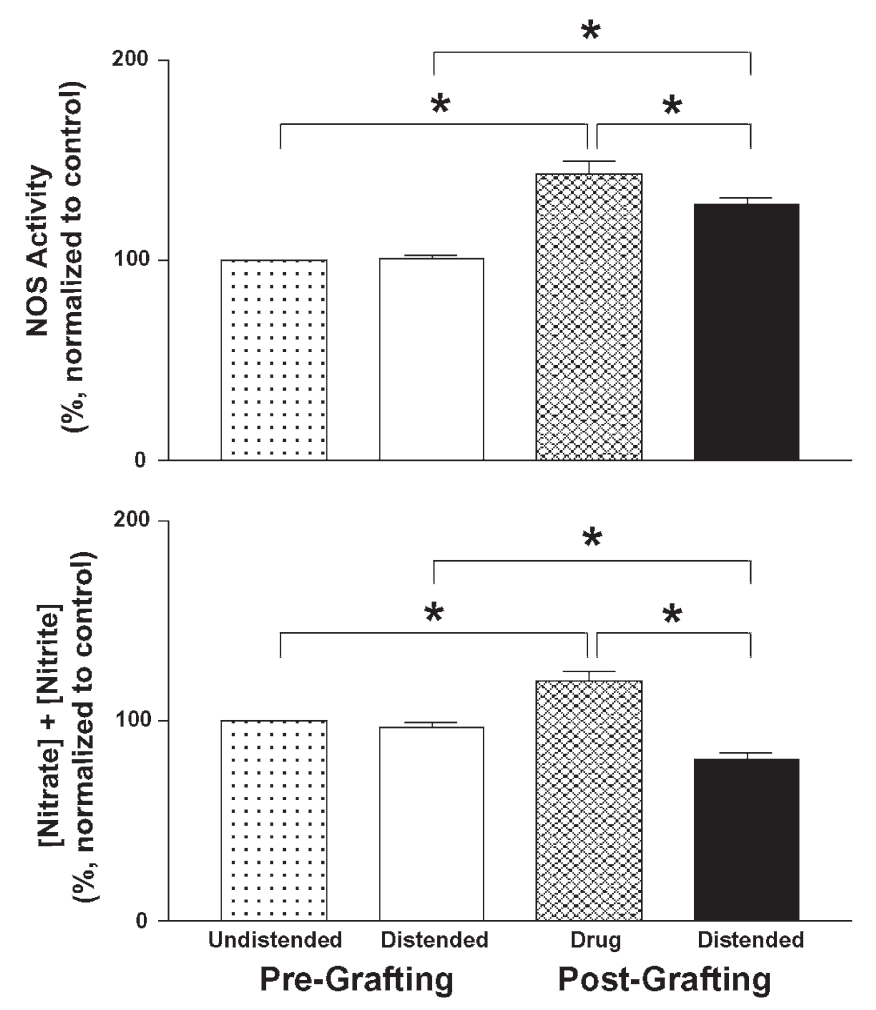

Figure 6. Enzymatic activities of nitric oxide synthase (NOS) and concentrations of nitrate and nitrite determined by using a colorimetric assay. Bar charts are the averaged NOS activities (upper panel) and the sum of concentrations of nitrate and nitrite (lower panel). ${ }^{*} P<.05$.

endothelial layer, the average density of endothelium did not differ between the distended and nondistended veins (Figure 3, $A-C$ ). The unchanged level of eNOS protein immediately after distention (Figure 5, A) confirmed the absence of the endothelial cell loss. However, the endothelial cells were still modified by distention. Although it is difficult to detect the absence of the relaxation stimulated with acetylcholine at such a low level of contraction (Figure 2, A), the decreased eNOS expression after 2 weeks of grafting reveals the original effect of distention on the endothelial cells. Thus moderate distension did not cause de-endothelialization but still modifies cells, resulting in prolonged detrimental effects.

Grafting for 2 weeks significantly increased the eNOS expression in the drug-treated grafts, while downregulating its expression in those that were pressure distended. Phosphorylation of eNOS $\operatorname{Ser}^{1177}$ was increased by $110 \%$ in the drug-treated grafts. These levels of expression and phosphorylation were at least 2-fold higher in the drug-treated grafts than in the distended grafts. eNOS levels have previously been found to be significantly increased in SV grafts compared with those seen in ungrafted veins, ${ }^{7}$ but the effect of distention was not examined in that study. The difference in the regulation of eNOS between 2 types of grafts was not attributable to change in gene transcription, so that the discrepancy between eNOS mRNA and eNOS protein demonstrates complex regulatory mechanisms at the posttranscriptional level, the posttranslational level, or both.

In contrast, the protein expression of iNOS was doubled after arterial implantation, regardless of the preparatory procedures of the veins before grafting, and this was highly correlated to the upregulated gene transcription (Figure 4). This indicated that the inflammatory response triggered by surgical trauma and arterial hemodynamics masked the effect of the initial distention. However, the upregulated iNOS levels could not compensate for the effect of decreased eNOS levels with respect to either NOS activity or NO production. The NOS activity and NO production were not changed in the veins immediately after distention but were significantly higher in the drug-treated grafts than in the distended grafts (Figure 6). Because NO has an extremely short half-life, its local distribution and subsequent effects largely depend on the NOS expression. Thus NOS activity and NO production principally reflected the total levels of eNOS plus iNOS expression.

This study characterized the NO regulation in the grafts after arterial implantation of the distended and nondistended veins. Cellular mechanisms of vascular remodeling, including NO regulation, have been studied more extensively in balloon-injured arteries than in bypass vein grafts. NO is known to inhibit platelet aggregation and expression of adhesion molecules, and this antiatherogenic property might reduce the period during which the vein graft is vulnerable to thrombosis. ${ }^{17-20}$ In late graft failure NO might play a role in the reduction of intimal hyperplasia because it is implicated in the attenuation of vascular SMC proliferation and migration $^{13-15}$ and the development of atherosclerosis. ${ }^{21}$ Furthermore, the higher NO formation in the internal thoracic artery compared with the SV underlies a better early and long-term patency of this conduit vessel over the SV in coronary bypass grafting (Figure 1). ${ }^{22}$ Our recent data revealed that the drug-treated grafts exhibited reduced neointima formation, which could be attributed to the increased levels of NO (Figure 6). Therefore the replacement of distention with pharmacologic relaxation, which results in higher levels of NO in the grafts, could contribute to the reduction in neointima development and possibly improved vein graft patency.

iNOS is known to be induced after injury and potently contributes to NO production. It has been identified within the media 1 day after balloon injury but was confined to neointimal SMCs by 1 to 2 weeks. ${ }^{23}$ It could compensate for the loss of $\mathrm{NO}$ as a result of endothelial damage and downregulated eNOS levels. ${ }^{24}$ However, eNOS and iNOS 
might differentially regulate vascular remodeling during vein graft failure. It has been suggested that NO from eNOS inhibited neointimal formation, whereas NO from iNOS suppressed the development of constrictive remodeling, defined as the reduction of the cross-sectional vascular area. ${ }^{25}$

The avoidance of distention resulted in a beneficial increase of NO production. In previous studies endothelial damage was observed after manual distention and flushing of vessels. ${ }^{89}$ However, we did not detect acute denudation of endothelial cells either in human veins ${ }^{4}$ or in the present study after distention of porcine veins. This was probably due to the moderate pressure and the absence of a flushingout rinse; nevertheless, the consequences of eNOS in the distended veins were still detected after grafting. It has been shown that human SVs harvested by means of either the minimally invasive technique or the traditional open dissection technique preserve the endothelial relaxation response and SMC contractile function if the veins were not subject to distention. ${ }^{26}$ Application of a no-touch harvesting technique of SVs, in which the vein is harvested with a pedicle of surrounding tissue and not distended, also preserved the endothelial integrity and function. ${ }^{6}$ NOS content was higher in the SVs harvested by this technique than in SVs harvested by the conventional procedure including distention, ${ }^{6}$ and distention is suggested to have a detrimental effect on luminal endothelial and medial NOS levels.

Pressure distention, unfortunately, cannot be avoided without replacement with an alternative technique to overcome vasospasm. We used a combination of pharmacologic vasodilatators, which appeared to be potently antispasmodic on human SVs. ${ }^{4}$ The pharmacologic cocktail might contribute to the effects observed in this study. For instance, the Rho-kinase inhibitor fasudil has been demonstrated to reverse hypoxiainduced downregulation of eNOS mRNA, protein expression, and activity in human SV endothelial cells. ${ }^{27}$

In conclusion, we demonstrated that pressure distention of veins during surgical preparation for grafting, compared with treating the veins with a combination of vasodilatory drugs, resulted in reduced eNOS expression and phosphorylation and low NOS activity and NO production in vein grafts after arterial implantation. This study supports the hypothesis that avoidance of pressure distention by using pharmacologic vasodilatation constitutes a feasible clinical technique for augmenting NO production by the vein graft and eventually improving graft patency.

The first limitation of this study is the relatively small number of experimental animals. However, our study was of sufficient statistical power to delineate differences in NOS expression and NO levels between the distended and drug-treated grafts. Furthermore, the conclusions of our study are based not on the analysis of a single variable but on several parameters (gene transcription, protein expression, activity, and NO level). Second, the assay kit for measuring NO concentration and NOS activity has a measuring limit at micromolar range. Compared with a porphyrinic nanosensor, which is able to directly measure $\mathrm{NO}$ at a nanomolar range of concentration, this colorimetric NO measurement assay is relatively insensitive, which is probably related to the indirect measurement of NO from the sum of the levels of nitrate and nitrite. In addition, using this kit does not allow one to distinguish the contributions of each NOS in the NO production.

We thank Mr Serge Okon for his help with image acquisition and analysis.

\section{References}

1. Motwani JG, Topol EJ. Aortocoronary saphenous vein graft disease: pathogenesis, predisposition, and prevention. Circulation. 1998;97: 916-31.

2. Shuhaiber JH, Evans AN, Massad MG, Geha AS. Mechanisms and future directions for prevention of vein graft failure in coronary bypass surgery. Eur J Cardiothorac Surg. 2002;22:387-96.

3. Crowley CM, Lee CH, Gin SA, Keep AM, Cook RC, Van Breemen C. The mechanism of excitation contraction coupling in phenylephrinestimulated human saphenous vein. Am J Physiol Heart Circ Physiol. 2002;283:H1271-81.

4. Okon EB, Millar MJ, Crowley CM, Bashir JG, Cook RC, Hsiang YN, et al. Effect of moderate pressure distention on the human saphenous vein vasomotor function. Ann Thorac Surg. 2004;77:108-14.

5. Bilfinger TV, Stefano GB. Human aortocoronary grafts and nitric oxide release: relationship to pulsatile pressure. Ann Thorac Surg. 2000;69:480-5.

6. Tsui JC, Souza DS, Filbey D, Karlsson MG, Dashwood MR. Localization of nitric oxide synthase in saphenous vein grafts harvested with a novel "no-touch" technique: potential role of nitric oxide contribution to improved early graft patency rates. J Vasc Surg. 2002;35:356-62.

7. Jeremy JY, Dashwood MR, Timm M, Izzat MB, Mehta D, Bryan AJ, et al. Nitric oxide synthase and adenylyl and guanylyl cyclase activity in porcine interposition vein grafts. Ann Thorac Surg. 1997;63:470-6.

8. Svendsen E, Dalen H, Moland J, Engedal H. A quantitative study of endothelial cell injury in aorto-coronary vein drafts. J Cardiovasc Surg. 1986;27:65-71.

9. Hofer H, Mihatsch MJ, Guggenheim R, Amsler B, Hasse J, Graedel E. Morphologic studies in saphenous vein grafts for aorto-coronary bypass surgery. Part I: Morphology of the Graft using ordinary surgical preparation techniques. Thorac Cardiovasc Surg. 1981;29:32-7.

10. Angelini GD, Bryan AJ, Williams HM, Morgan R, Newby AC. Distention promotes platelet and leukocyte adhesion and reduces shortterm patency in pig arteriovenous bypass grafts. $J$ Thorac Cardiovasc Surg. 1990;99:433-9.

11. Chung AW, Rauniyar P, Luo H, Hsiang YN, van Breemen C, Okon EB. Pressure distention compared with pharmacologic relaxation in vein grafting upregulates matrix metalloproteinase-2 and -9. J Vasc Surg. 2005;42:747-56.

12. Duda DG, Fukumura D, Jain RK. Role of eNOS in neovascularization: NO for endothelial progenitor cells. Trends Mol Med. 2004;10:143-5.

13. Anderson PG, Boerth NJ, Liu M, McNamara DB, Cornwell TL, Lincoln TM. Cyclic GMP-dependent protein kinase expression in coronary arterial smooth muscle in response to balloon catheter injury. Arterioscler Thromb Vasc Biol. 2000;20:2192-7.

14. von der Leyen HE, Gibbons GH, Morishita R, Lewis NP, Zhang L, Nakajima M, et al. Gene therapy inhibiting neointimal vascular lesion: in vivo transfer of endothelial cell nitric oxide synthase gene. Proc Natl Acad Sci U S A. 1995;92:1137-41.

15. Shears LL 2nd, Kibbe MR, Murdock AD, Billiar TR, Lizonova A, Kovesdi I, et al. Efficient inhibition of intimal hyperplasia by adeno- 
virus mediated inducible nitric oxide synthase gene transfer to rats and pigs in vivo. J Am Coll Surg. 1998;187:295-306.

16. Bank AJ. Physiologic aspects of drug therapy and large artery elastic properties. Vasc Med. 1997;2:44-50.

17. Radomski MW, Palmer RM, Moncada S. An L arginine/nitric oxide pathway present in human platelets regulates aggregation. Proc Natl Acad Sci U S A. 1990;87:5193-7.

18. Chung AW, Wildhirt SM, Wang SH, Koshal A, Radomski MW. Combined administration of nitric oxide gas and iloprost during cardiopulmonary bypass reduces platelet dysfunction: a pilot clinical study. J Thorac Cardiovasc Surg. 2005;129:782-90.

19. Landmesser U, Harrison DG, Drexler H. Oxidant stress-a major cause of reduced endothelial nitric oxide availability in cardiovascular disease. Eur J Clin Pharmacol. 2005;12:1-7.

20. Murohara T, Witzenbichler B, Spyridopoulos I, Asahara T, Ding B, Sullivan A, et al. Role of endothelial nitric oxide synthase in endothelial cell migration. Arterioscler Thromb Vasc Biol. 1999;19:1156-61.

21. Melichar VO, Behr-Roussel D, Zabel U, Uttenthal LO, Rodrigo J, Rupin A, et al. Reduced cGMP signaling associated with neointimal proliferation and vascular dysfunction in late-stage atherosclerosis. Proc Natl Acad Sci U S A. 2004;101:16671-6.
22. Luscher TF, Diederich D, Siebenmann R, Lehmann K, Stulz P, von Segesser L, et al. Difference between endothelium-dependent relaxation in arterial and in venous coronary bypass grafts. $N$ Engl J Med. 1988;319:462-7.

23. Yan Z, Hansson GK. Overexpression of inducible nitric oxide synthase by neointimal smooth muscle cells. Circ Res. 1998;82:21-9.

24. Cuzzocrea S, Chatterjee PK, Mazzon E, Dugo L, De Sarro A, Van de Loo FA, et al. Role of induced nitric oxide in the initiation of the inflammatory response after postischemic injury. Shock. 2002;18: 169-76.

25. Yogo K, Shimokawa H, Funakoshi H, Kandabashi T, Miyata K, Okamoto S, et al. Different vasculoprotective roles of NO synthase isoforms in vascular lesion formation in mice. Arterioscler Thromb Vasc Biol. 2000;20:E96-100.

26. Black EA, Guzik TJ, West NE, Campbell K, Pillai R, Ratnatunga C, et al. Minimally invasive saphenous vein harvesting: effects on endothelial and smooth muscle function. Ann Thorac Surg. 2001;71:1503-7.

27. Takemoto M, Sun J, Hiroki J, Shimokawa H, Liao JK. Rho-kinase mediates hypoxia-induced downregulation of endothelial nitric oxide synthase. Circulation. 2002;106:57-62. 